\title{
The impact of oxidative stress on binding of drugs with plasma proteins studied by fluorescence anisotropy methods
}

\author{
Tomasz Wybranowski, Blanka Ziomkowska, Michał Cyrankiewicz and Stefan Kruszewski \\ Medical Physics Division, Biophysics Department, Collegium Medicum of Nicolaus Copernicus University, Bydgoszcz, Poland
}

\begin{abstract}
The aim of this study was to investigate the effect of oxidative stress on drug binding affinity, free and bound fraction. The fluorescence anisotropy method was used to examine the interactions of commercial human serum albumin (HSA) and human plasma proteins with ochratoxin A (OTA) or carboxylate form of camptothecin (CPT-C). In-vitro method revealed significant reduction in bound fraction of drugs to HSA oxidized by chloramine T. Oxidative stress was determined by measuring the plasma level of advanced oxidation protein products (AOPP). A significant positive correlation between the AOPP and the plasma free fraction of tested drugs in thirty healthy patients was also found. The oxidative stress monitoring by AOPP is very important for improvement of dosage of drugs highly bound to albumin and with narrow therapeutic index. As a result of severe oxidative stress, the drug pharmacokinetics and therapeutic effects are prone to change. This study highlights the issues of therapeutic drug monitoring and it can explain the behaviour of drugs in pathological situations.
\end{abstract}

Key words: Binding affinity - AOPP - Oxidative stress - Camptothecin - Ochratoxin A Albumin

\section{Introduction}

Interactions with proteins play an important role in the transport and action of drugs, toxins and other biologically active compounds due to the following reasons: (1) only free fraction of drug is pharmacologically active, (2) the therapeutic effect is closely related to free fraction of drug, (3) binding of drug has an effect on biological halflife, metabolism and excretion of drug (Sudlow et al. 1975; Kragh-Hansen et al. 2002). Binding of ligands to proteins was first reported in 1945 and referred to penicillin derivatives (Tompsett et al. 1945; Klotz et al. 1950). Researchers have found that its derivatives bind to proteins to varying degrees. A few years later, it was demonstrated that most drugs bind primarily to albumin, and the binding affects their bioavailability and toxicity in the body. The pharmacological effect depends on the ability of the drug penetration into tissue, where its action is to be started (Kober et al. 1980). The aim of a dose-adjustment is to achieve an optimal efficacy with-

Correspondence to: Tomasz Wybranowski, Medical Physics Division, Biophysics Department, Collegium Medicum of Nicolaus Copernicus University, Jagiellońska St. 13, 85-067 Bydgoszcz, Poland E-mail: tomaszwybranowski@cm.umk.pl out toxic side effects, according to the maxim of Paracelsus, a sixteenth-century German alchemist and doctor, i.e.: "All things are poison and nothing is poison, it is only the dose which makes a thing poison". Evaluating a degree of drug binding to protein is helpful in explaining the pharmacological effect of therapeutic agents (Dasgupta 2008).

An excessive free radical formation and a reduced activity of antioxidant systems (oxidative stress) cause an oxidative damage of proteins, especially under pathological conditions (Berlett et al. 1997; Iwao et al. 2006; Kawakami et al. 2006; Sitar et al. 2013). According to literature, there are only few studies examining the effects of albumin oxidation by free radicals on the affinity of drugs to albumin (Kurz et al. 1977; Wörner et al. 1990; Meucci et al. 1991; Burczynski et al. 1995; Anraku et al. 2001, 2004; Oettl et al. 2007). These tests are mainly based on the assessment of drugs binding to a commercial albumin. Most of the available studies on the binding properties of oxidized albumin were performed using in vitro albumin oxidized by hydrogen peroxide, chloramine $\mathrm{T}$, glucose, cysteine, homocysteine, nitrous oxide and ascorbic acid in presence of $\mathrm{FeCl}_{2}$. The following methods were used to study the affinity: ultrafiltration, dialysis or high-performance liquid chromatography (HPLC). 
Steady-state fluorescence spectroscopy is an important and widely used technique in physical sciences and is very useful for the study of the behaviour of molecules in solutions containing albumin (Wybranowski et al. 2008, 2014). The interaction between ligands and human serum albumin (HSA) under physiological conditions was investigated by the fluorescence anisotropy method. Fluorescence anisotropy is a useful tool in pharmacokinetics, and can be successfully applied in determining binding affinity constant of drug to protein (Kruszewski et al. 2006). The proposed method provides a possibility of quick, non-invasive study of biological blood plasma samples with high repeatability of measurements. The tested systems interact only with low intensity UV-VIS radiation. This method does not require additional reagents. It eliminates the need for dissolution of the tested solution samples in mobile phase solvents, what enables the measurement under physiological conditions. Compared to other methods, the presented one provides a more accurate measurement of free fraction of drug.

In an experimental model, albumin was oxidized by chloramine $\mathrm{T}$ due to its similarity to the oxidized albumin in vivo. Chloramine $\mathrm{T}$ is formed in the body as a result of reaction initiated by superoxide anion produced by phagocytes. In the presence of halide ions and under the myeloperoxidase, the superoxide anion undergoes dismutation to hydrogen peroxide leading to the formation of hypochlorous acid. Chloramine $\mathrm{T}$ is produced in the reaction of hypochlorous acid with ammonia and protein amino groups (Anraku 2003; Piwowar 2010). It is highly reactive.

Two drugs, ochratoxin A (OTA) and camptothecin (CPT), have been chosen for this study because of their high affinity to albumin (bound drug fraction > 99\%) and superior fluorescence properties. OTA is a toxic chemical substance produced by certain species of moulds growing on some food products, particularly cereals. Determining binding intensity of OTA to albumin is helpful in explaining the effects of toxic influence of OTA (Wybranowski et al. 2014). CPT is a natural product isolated in the early 1960s from the Chinese tree Camptotheca acuminata and exhibits antitumor activity. There are two forms of CPT (the carboxylate form and the lactone form), which exist in different conditions. Only carboxylate form (CPT-C) binds effectively to HSA (Burke et al. 1994).

\section{Materials and Methods}

\section{Chemicals}

OTA and HSA were received from Sigma-Aldrich. Stock solution of OTA ( $2 \mathrm{mM}$ ) was prepared in ethanol. The albumin was diluted in phosphate-buffered saline (PBS) at $\mathrm{pH}$ 7.4. The sample of CPT and topotecan was obtained from the Laboratory of Biotechnology, College of Pharmacy, University of Kentucky, Lexington, USA. $2 \mathrm{mM}$ stock solution of CPT lactone was prepared in DMSO (dimethylsulfoxide $\mathrm{C}_{2} \mathrm{H}_{6} \mathrm{OS}$ ). Such stock solution contains only a pure lactone form (CPT-L). $1 \mathrm{mM}$ stock CPT-C solution was obtained by dilution of stock CPT-L solution in PBS at $\mathrm{pH} 10$ in volume ratio 1:1. The concentration of CPT-C and OTA in final samples used for fluorescence anisotropy measurements was equal to $1 \mu \mathrm{M}$.

\section{Measurements in pure albumin}

Measurement of drug fluorescence anisotropy was performed for 8 albumin concentrations (in $\mu \mathrm{M}: 0.1,0.2,0.5$, $1,2,5,10,20)$ immediately after adding OTA and CPT-C to albumin solution and mixing the sample for $30 \mathrm{~s}$. Steadystate anisotropy of CPT carboxylate diluted in $20 \mu \mathrm{M}$ HSA solution instantaneously after mixing achieves maximum value and stay constant over time (Kruszewski at al. 2008).

\section{Plasma measurements}

The human blood from healthy people was received from the Local Blood-Donation Center (Bydgoszcz, Poland). Thirty healthy patients were enrolled in this study. The test group was drawn out of men and women in the age of 18 to 65 years. $8 \mathrm{ml}$ of blood sample was taken for examination from each patient. Blood samples were added to standard sterile polystyrene tubes containing EDTA and then centrifuged at $4000 \mathrm{rpm}$ for 5 minutes. The concentration of albumin in each sample was determined by colorimetric method. At pH 3.6 a colored complex of albumin with bromocresol green is formed. The absorbance of the solution measured at wavelength $630 \mathrm{~nm}$ is proportional to the concentration of albumin in the sample. After estimating albumin concentration in the patient sample, 8 different dilutions of each sample in PBS depending on albumin concentration (in $\mu \mathrm{M}: 0.1,0.2,0.5,1,2,5,10,20$ ) were prepared. The single fluorescence anisotropy measurement after adding OTA and CPT-C to plasma solution and mixing the sample for $30 \mathrm{~s}$ was recorded. The final concentration of OTA and CPT-C was equal to $1 \mu \mathrm{M}$.

The level of AOPP was determined by measuring absorbance at $340 \mathrm{~nm}$ according to the modified method described for the first time by Witko-Sarsat (Witko-Sarsat et al. 1996; Hanasand et al. 2012). Briefly, the reactant mixture for AOPP assay contains $1.875 \mathrm{ml}$ of $0.2 \mathrm{M}$ citric acid and $25 \mu \mathrm{l}$ of $1.16 \mathrm{M}$ potassium iodide. Then $1.9 \mathrm{ml}$ of this mixture was added to $100 \mu \mathrm{l}$ of the test sample and after $30 \mathrm{~min}$ the absorbance was recorded. The results were expressed as chloramine $\mathrm{T}$ equivalents and divided by concentration of proteins. In comparison to the original method, citric acid was used instead of acetic acid. This modified method is characterized by greater stability over time. 


\section{Preparation of oxidized albumin}

The investigation of the effects of chloramine $T$ on binding affinity of tested drugs, involved preparation of 9 different ratios of chloramine $\mathrm{T}$ and albumin $(0,1,2,3,4,5,10$, $15,30)$. The concentration of albumin in each sample was $100 \mu \mathrm{M}$. Then, 8 different concentrations of each solutions with various concentrations of albumin (in $\mu \mathrm{M}: 0.1,0.2$, $0.5,1,2,5,10,20)$ were prepared. The next step was to add OTA or CPT-C to sample (final concentration of OTA and CPT-C was equal to $1 \mu \mathrm{M}$ ) and fluorescence anisotropy was recorded.

\section{Anisotropy measurements}

A PTI (Photon Technology International, Birmingham, NJ, USA) spectrofluorometer was used for the measurement of steady-state fluorescence anisotropy. Measurements of fluorescence anisotropy of drugs in HSA solution were performed with the instrument in the "L-format" using excitation at $370 \mathrm{~nm}$ and $420 \mathrm{~nm}$ long-pass filters on the emission channel. The use of long pass filter on emission channel ensures the separation of fluorescence from scattered light. Both excitation and emission channels were equipped with computer-driven motorized polarising filters. The emission maxima of unbound CPT-C and OTA are at $447 \mathrm{~nm}$ and $448 \mathrm{~nm}$, respectively. HSA does not absorb light at $370 \mathrm{~nm}$. The temperature of the sample was kept constant $\left(37^{\circ} \mathrm{C}\right)$ using the ultrathermostat TW2.03 (ELMI).

As a consequence of random orientation of molecules, their rotational movements and limited lifetime of the excited (electronic) states of fluorophores the fluorescence light emitted by the solution is always depolarized. For the characterization of depolarization degree, the anisotropy $r$ is usually used. It is defined as follows:

$r=\frac{I_{V}-G I_{H}}{I_{V}+2 G I_{H}}$

where $I_{V}$ and $I_{H}$ are the fluorescence intensities of the vertically and horizontally polarized emission, when the sample is excited by vertically polarized light. The coefficient $G=$ $S_{V} / S_{H}$ is the ratio of the sensitivities $S$ of the detection system for vertically and horizontally polarized light. During measurement of the sensitivities, the excitation polarization was kept horizontal. Due to photoselection and internal conversion, the maximum possible values of anisotropy of fluorescence light emitted by a solution is always smaller than 0.4 (Lakowicz 1999). Rotation of fluorescent molecules during the life of an excited state causes further decrease of anisotropy. The fluorescence anisotropy methods can be used for determining the concentration of bound drug in relation to the HSA concentration. Fluorescence anisotropy of bound drug is close to 0.3 as a result of slow rotation. A free drug can fast rotate and its anisotropy is small (close to 0 ). The total fluorescence anisotropy $r$ observed for mixture of fluorophores is given by:

$r=\sum_{i} r_{i} f_{i}$

where $r_{i}$ is the anisotropy of $\mathrm{i}$-th fluorophore and $f_{i}$ is the fraction of the total fluorescence intensity provided by $\mathrm{i}$-th fluorophore. If fluorescent drug is dissolved in a protein solution, two kinds of fluorophores exist: free molecules and molecules bound to proteins. Moreover, taking into account the influence of scattered light, the experimentally determined anisotropy is given as:

$r=\mathrm{r}_{\mathrm{F}} \mathrm{f}_{\mathrm{F}}+\mathrm{r}_{\mathrm{B}} \mathrm{f}_{\mathrm{B}}+\mathrm{r}_{\mathrm{S}} \mathrm{f}_{\mathrm{S}}$

where $r_{F}$ and $r_{B}$ are the fluorescence anisotropies of the free and bound drugs, respectively, and $f_{F}$ and $f_{B}$ are their fractional fluorescence intensity, $r_{S}$ is the anisotropy of pure HSA solution and $f_{S}$ its fraction in the total light intensity provided by samples and reached the photodetector. The scattering originating from drug molecules is negligible in the presented case.. The sum of the above light intensities fractions equals 1 . However, due to very high selectivity of the used optical filters, the fraction of light originated from scattering processes is also extremely low, so

$f_{B}=\frac{r-r_{F}+f_{S} \cdot\left(r_{F}-r_{S}\right)}{r_{B}-r_{F}} \approx \frac{r-r_{F}}{r_{B}-r_{F}}$

The fluorescence anisotropy of free drug $r_{F}$ is obtained by anisotropy measurement in solution free of proteins. The ordinate intercept of regression line 1/r on 1/HSA (where HSA represents the concentration of HSA) can be interpreted as inverse of the fluorescence anisotropy $r_{B}$. On the basis of experimentally determined $r, r_{F}$ and estimated value of $r_{B}$, the fraction of fluorescence intensity $f_{B}$ can be calculated from the above equation.

The fractional fluorescence intensities $f_{F}$ and $f_{B}$, are proportional to the concentration of free $\left(C_{F}\right)$ and bound $\left(C_{B}\right)$ drug, respectively. A quantum yield of fluorescence of examined drugs may change upon binding with proteins, so the following approximate relations occur:

$C_{F} \propto f_{F} / I_{C, O}$ and $C_{B} \propto f_{B} / I_{C, H S A m a x}$

where $I_{C, 0}$ is the fluorescence intensity of the solution of pure (and also free) drug and $I_{C, H S A m a x}$ is the maximum fluorescence intensity of drug upon binding to albumin, where all drug molecules are bound to protein. Due to the relation between free drug fraction $\left(F_{B}\right)$ and free drug concentration:

$F_{B}=C_{B} / C_{T}=C_{B} /\left(C_{B}+C_{F}\right)$ 
where $C_{T}$ is total concentration of drug, the quantum yieldcorrected Eq. (6) can be rewritten into

$F_{B}=\frac{1}{g \cdot \frac{f_{F}}{f_{B}}+1}$

where $g$ is the ratio of maximum fluorescence intensities of drug upon binding to albumin and free forms of drug (quantum yield enhancement factor).

By substituting Eq. (4) into Eq. (7), the following formula can be obtained:

$F_{B}=\frac{r-r_{F}}{r-r_{F}+g\left(r_{B}-r\right)}$

On the basis of Eq. (8) one can estimate fraction of bound ligand also in the case of changing quantum yield (Lakowicz 1999; Jameson et al. 2010).

\section{Binding affinity constant calculation}

For determining the binding constant $(K)$ of ligand to HSA, the following formula was used (Lakowicz 1999):

$\frac{H S A_{B}}{H S A_{T}}=\frac{K \cdot C_{F}}{1+K \cdot C_{F}}$

where $H S A_{T}=H S A_{F}+H S A_{B}$ is the total concentration of HSA, $H S A_{B}$ is the concentration of bound albumin, while $C_{F}$ and $H S A_{F}$ are concentrations of free ligand and free albumin, respectively. Because $C_{F}=F_{F} \cdot C_{T}$ and $C_{B}=$ $F_{B} \cdot C_{T}$. Eq. (9) can be rearranged yielding the modified Scatchard equation:

$\frac{F_{B}}{H S A_{T} \cdot\left(1-F_{B}\right)}=-\frac{F_{B} \cdot C_{T}}{H S A_{T}} \cdot K+K$

where $F_{B}$ and $F_{F}$ are the fraction of ligand bound and unbound to human serum albumin respectively. The affinity constant can be calculated by determining a slope or intercept of the regression line. Coefficients of correlation and standard errors of slopes and intercepts were calculated with the use of Origin 7.0 software application. Then, standard error of the binding constant $\mathrm{K}$ was estimated.

The percentage of bound and free fraction of drug calculation in plasma

If the binding affinity constant $(K)$ is known it is possible to determine the theoretical percentage of bound and free fraction of drug for the physiological concentration of albumin in each patient. Assuming that $H S A_{T B}>>C_{T}$, the percentage of bound $\left(C_{B \%}\right)$ and free fraction $\left(C_{F \%}\right)$ of drug can be calculated according formula as follows (Kratochwil 2002):
$C_{B \%}=\frac{H S A_{T B}}{H S A_{T B}+1 / K} \cdot 100 \%$

$C_{F \%}=100 \%-C_{B \%}$

where $H S A_{T B}$ is the concentration of albumin in blood of individual patients. Actually, in the blood the concentration of drug is usually much smaller than physiological concentration of albumin. The percentage of free fraction is usually independent of total concentration of drugs in the range of their therapeutic concentrations. It was also assumed that a binary complex is formed.

The percentage of bound and free fraction of drug calculation in pure albumin

The free and bound fraction were calculated on the basis of Eq. $(11,12)$ for the albumin concentration of $640 \mu \mathrm{M}$. This value was selected based on albumin concentration in plasma for 30 patients and is the mean of these measurements.

\section{Results}

\section{Measurements in pure albumin}

As a result of binding of the drugs and albumin, the possibility of rotational motion of molecules significantly decreases and the value of anisotropy increases greatly in a function of albumin concentration. Rate of increase of fluorescence anisotropy over HSA concentration determines qualitatively a binding efficiency of drugs to HSA. The scattered emission light by proteins within the range of tested protein concentrations does not also affect the measured fluorescence anisotropy value of the studied drugs. The statement can be evidenced by measurements performed for non-albumin-binding drug, e.g. topotecan. The fluorescence anisotropy of $2 \mu \mathrm{M}$ topotecan equals 0.024 if measured in $20 \mu \mathrm{M}$ albumin solution and 0.025 in the absence of albumin. To explore whether the scattered light interfere with anisotropy measurements, an experiment was conducted in which OTA and CPT-C were excited and then the emission light was passing through a sample of $20 \mu \mathrm{M}$ albumin solution (albumin sample was not excited). In these cases, no change in fluorescence anisotropy is observed (0.025 vs. 0.027 for OTA and 0.031 vs. 0.029 for CPT-C). The two experiments show unequivocally that scattering of emission light on albumin molecules does not alter the fluorescence anisotropy of the studied drugs. The light at the emission wavelength is also low scattered and absorbed by albumin. The value of absorbance of $20 \mu \mathrm{M}$ albumin is equal 0.052 at the wavelength of $450 \mathrm{~nm}$. So, the inner filter effect does not influence on observed fluores- 


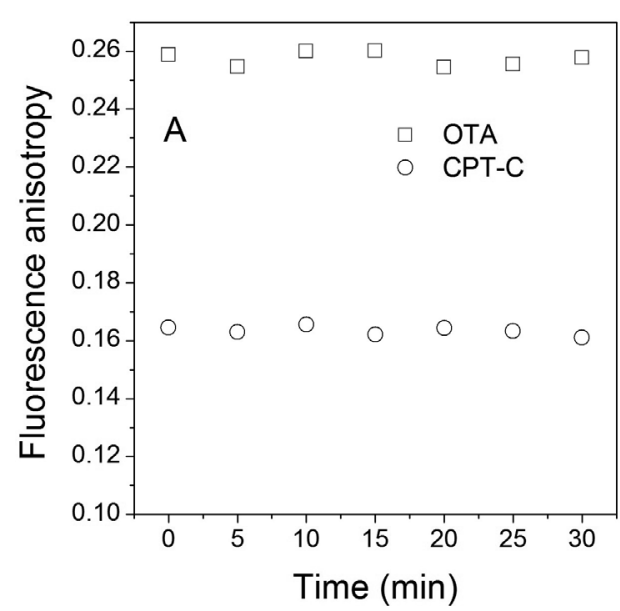

Figure 1. Time dependence of fluorescence anisotropy (A) and intensity (B) of camptothecin (CPT-C) and ochratoxin A (OTA) after $30 \mathrm{~s}$ mixing with $5 \mu \mathrm{M}$ human serum albu$\min$ (HSA). cence intensity of drug. Fig. 1 shows changes in the steadystate anisotropy and fluorescence intensity signal of CPT-C and OTA after $30 \mathrm{~s}$ mixing with $5 \mu \mathrm{M}$ HSA in time. The values stay constant over time. The situation is the same for other HSA concentrations. After binding of the investigated drugs with albumin, no change in wavelength of maximum emission was observed. However, increased fluorescence of OTA in dependence on concentration of albumin was noted. To calculate $\mathrm{g}$ factor the intensity of fluorescence of free OTA and at saturation regime (albumin concentration $20 \mu \mathrm{M}$ ) were measured. Changes in fluorescence intensity were included in $F_{B}$ estimates according to Eq. (8). On the basis of the measured values of fluorescence anisotropy of OTA and CPT-C in albumin solution it can be concluded that OTA binds stronger to albumin than CPT-C (Fig. 2 ). At the albumin concentration of $2 \mu \mathrm{M}$, fluorescence anisotropy values of OTA and CPT-C equal $0.25,0.11$, respectively. By plotting the values $F_{B} / H S A_{T}\left(1-F_{B}\right)$ vs. $F_{B} C_{T}$ ' $H S A_{T}$, a straight line was obtained (Fig. 3). Its intersection with the axis of ordinates gives, according to Eq. (10), the value of affinity constant of drug. The determined affinity constant of OTA to albumin equals $5237 \pm 105 \mathrm{mM}^{-1}$. The affinity of the carboxylate form of CPT is much smaller and equals $292 \pm 11 \mathrm{mM}^{-1}$. Estimated values of free fraction of OTA and CPT-C in blood are respectively $0.029 \%$ and $0.51 \%$, according to Eq. $(11,12)$.

\section{Effect of chloramine T on binding properties of pure albumin}

To investigate the effects of free radicals on the binding of drugs to albumin, HSA was oxidized by chloramine T. Fig. 4 shows the resulting affinity constant of OTA and $\mathrm{CPT}-\mathrm{C}$ as a function of ratio of chloramine $\mathrm{T}$ and albumin $\left(\mathrm{C}_{\mathrm{CH}} / \mathrm{C}_{\mathrm{HSA}}\right)$. As seen in the figure, the affinity of CPT-C and OTA clearly decreases with increasing concentration ratio $\mathrm{C}_{\mathrm{CH}} / \mathrm{C}_{\mathrm{HSA}}$. Table 1 shows how the affinity constants and the bound and free fractions of OTA and CPT-C depend on the amount of chloramine $\mathrm{T}$ in the solution. At the ratio $\mathrm{C}_{\mathrm{CH}} / \mathrm{C}_{\mathrm{HSA}}$ equals five, there is a significant increase in free fraction levels of OTA and CPT-C, namely approximately five and four-fold, respectively. That means that the degradation of albumin causes a marked increase in the level of the active part of a ligand responsible for the pharmacological effect.

\section{Measurements in plasma}

OTA and CPT-C fluorescence anisotropy measurements in function of albumin concentration for 30 plasma samples were recorded. Anisotropy measurements were performed for 8 dilutions of each patient sample prepared according

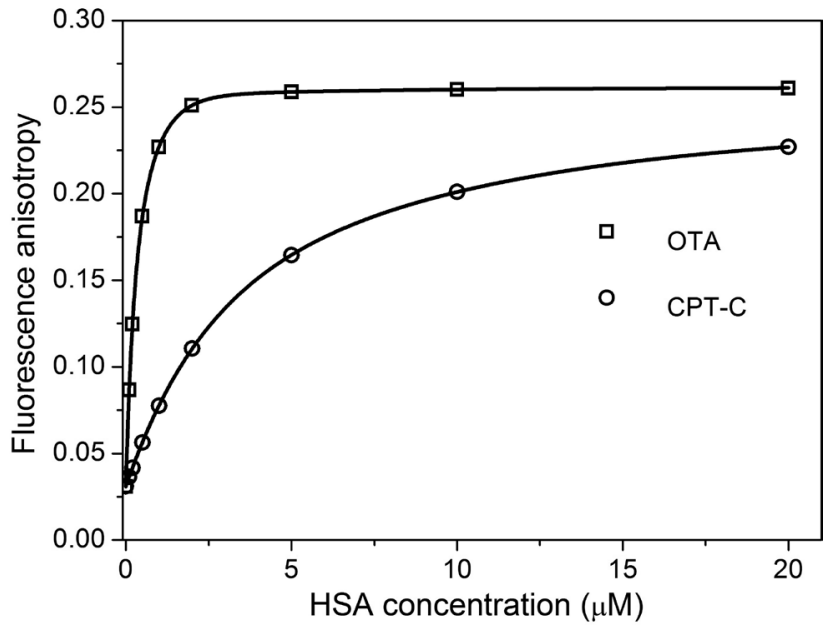

Figure 2. Fluorescence anisotropy of OTA and CPT-C depending on HSA concentration. For abbreviations, see Fig. 1. 


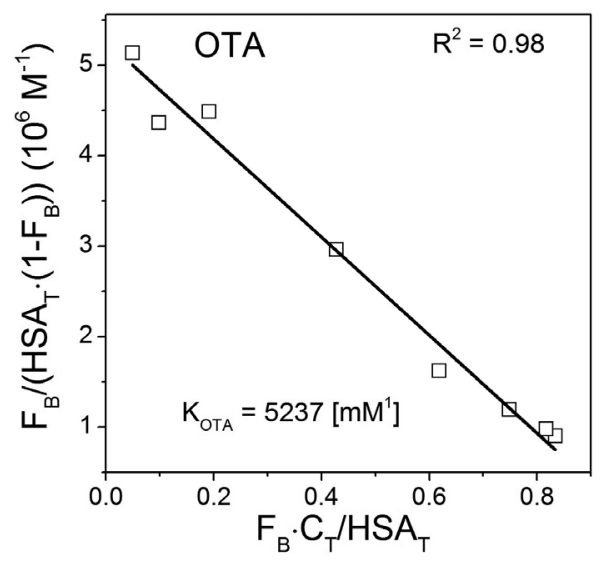

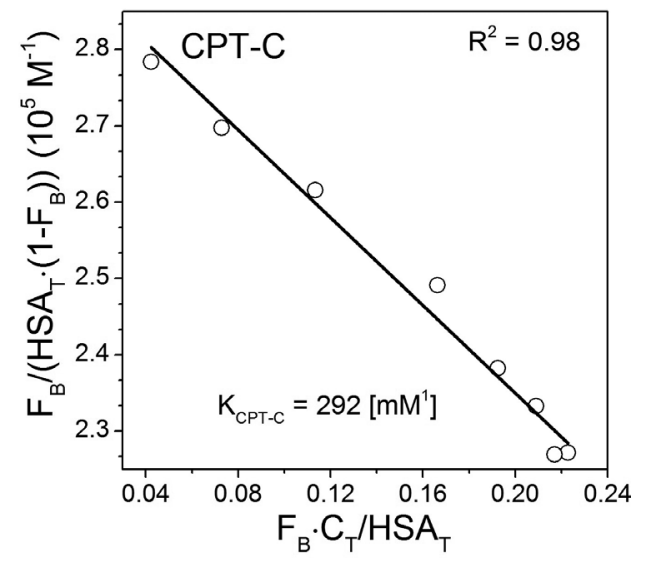

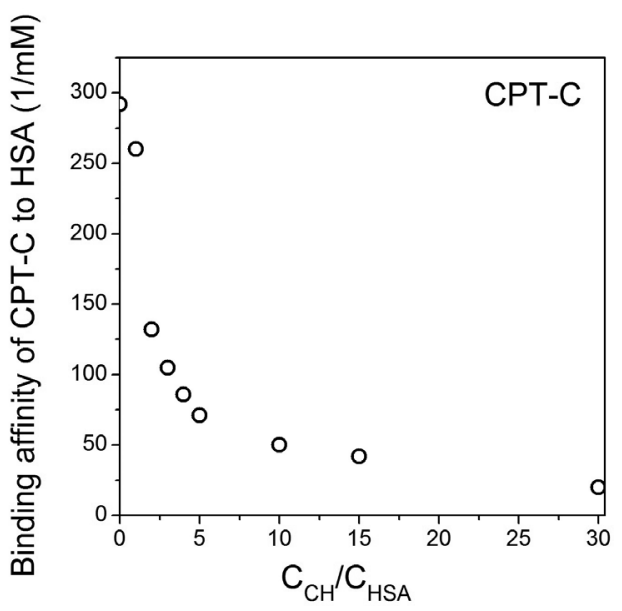

Figure 3. The curve used to estimate binding affinities of drugs for pure albumin according to Eq. (10). For abbreviations, see Fig. 1.

Figure 4. Binding affinity of OTA and CPT-C in dependence on ratio of chloramine $\mathrm{T}$ concentration $\left(\mathrm{C}_{\mathrm{CH}}\right)$ and HSA concentration $\left(\mathrm{C}_{\mathrm{HSA}}\right)$. For abbreviations, see Fig. 1. to albumin concentration. The increase of fluorescence anisotropy with increasing albumin concentration in patient samples is very similar to that obtained for pure albumin (data not shown). Figure 5 shows graph used to estimate binding affinities of CPT-C and OTA for measurements in plasma for representative sample. The obtained linear dependence shows suitability of application of the used model. On the basis of the measured values, the binding af-

Table 1. The binding affinity constants $(\mathrm{K})$ and the bound $\left(\mathrm{C}_{\mathrm{B} \%}\right)$ and free $\left(\mathrm{C}_{\mathrm{F} \%}\right)$ fractions of OTA and $\mathrm{CPT}-\mathrm{C}$ depending on ratio of chloramine $\mathrm{T}$ and HSA concentration $\left(\mathrm{C}_{\mathrm{CH}} / \mathrm{C}_{\mathrm{HSA}}\right)$

\begin{tabular}{lccccccc}
\hline \multirow{2}{*}{$\mathrm{C}_{\mathrm{CH}} / \mathrm{C}_{\mathrm{HSA}}$} & \multicolumn{3}{c}{$\mathrm{OTA}$} & & \multicolumn{3}{c}{$\mathrm{CPT}-\mathrm{C}$} \\
\cline { 2 - 3 } \cline { 7 - 8 } \cline { 7 - 8 } & $\mathrm{K}\left(\mathrm{mM}^{-1}\right)$ & $\mathrm{C}_{\mathrm{B} \%}(\%)$ & $\mathrm{C}_{\mathrm{F} \%}(\%)$ & & $\mathrm{K}\left(\mathrm{mM}^{-1}\right)$ & $\mathrm{C}_{\mathrm{B} \%}(\%)$ & $\mathrm{C}_{\mathrm{F} \%}(\%)$ \\
\hline 0 & 5237 & 99.971 & 0.029 & & 292 & 99.49 & 0.51 \\
2 & 4700 & 99.968 & 0.032 & & 260 & 99.42 & 0.58 \\
3 & 2541 & 99.941 & 0.059 & & 132 & 98.87 & 1.13 \\
4 & 2300 & 99.934 & 0.066 & & 105 & 98.59 & 1.41 \\
5 & 1300 & 99.884 & 0.116 & & 86 & 98.28 & 1.72 \\
10 & 1100 & 99.863 & 0.137 & & 71 & 97.92 & 2.08 \\
15 & 639 & 99.765 & 0.235 & & 50 & 97.08 & 2.92 \\
30 & 431 & 99.651 & 0.349 & & 42 & 96.54 & 3.46 \\
\hline & 139 & 98.927 & 1.073 & & 20 & 93.01 & 6.99 \\
\hline
\end{tabular}

OTA, ochratoxin A; CPT-C, camptothecin; HSA, human serum albumin. 

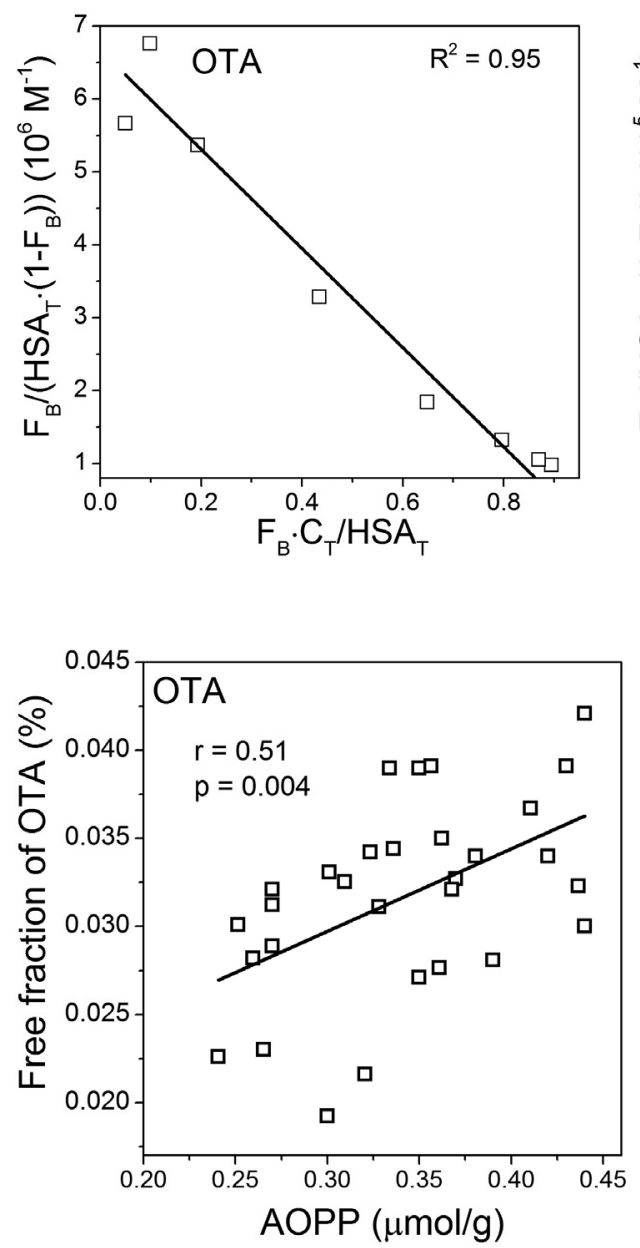

Figure 5. The curve used to estimate binding affinities of drugs for patient according to Eq. (10). For abbreviations, see Fig. 1.

Figure 6. Correlation between free fraction of OTA and CPT-C with advanced oxidation protein products (AOPP) in 30 healthy subjects. For more abbreviations, see Fig. 1.

finities of drugs were estimated. The binding affinities were converted into the percentage of free fraction according to Eq. $(11,12)$. Mean free fractions of OTA and CPT-C levels, calculated for all samples are $0.032 \%$ and $0.5 \%$ respectively. The level of AOPP was determined by spectrophotometric method for each plasma sample. As is apparent from Fig. 6, there are significant positive correlations between AOPP and free fraction of CPT-C $(r=0.51, p=0.004)$ and OTA $(r=$ $0.56, p=0.001)$. The fitting trendline indicates that a twofold increase in the degree of protein oxidation causes an increase in the free fraction of CPT-C and OTA by $35 \%$ and $37 \%$, respectively.

\section{Discussion}

Knowledge about drug interactions with proteins enables a proper dosing adjustment. The therapeutic effect is determined by an unbound fraction (Levy et al. 1984; Routledge et al. 1985; Wright et al. 1996; Akhlaghi et al. 1998; Dasgupta 2008; Krasowski et al. 2012). The determination of free drug blood concentrations is critical in controlling a safe drug level and achieving a high therapeutic efficacy. Currently, in most cases, the free fraction of drug is calculated on the basis of the affinity constant determined for commercial albumin (pure albumin) (Cohen 2004). This approach, although straightforward, does not allow to estimate precisely the level of free fraction for a particular patient. The reason is that free radicals lead to significant changes in properties of proteins but there are very little studies reporting connection between drug-albumin affinity and oxidative stress (Oettl et al. 2007). The understanding of this mechanism can be very useful in clinical practice and may help improve the efficacy and the individualization of the therapy. Most of the studies were performed using in vitro albumin oxidized by various oxidants. Anaruk et al. (2001) compared the binding affinity of drug to albumin mildly oxidized by ascorbic acid/ $/ \mathrm{FeCl}_{2}$ system, chloramine $\mathrm{T}$ or $\mathrm{H}_{2} \mathrm{O}_{2}$. They revealed a decrease in binding affinity of flurbiprofen. In the case of warfarin, mild oxidation of HSA had no effect on the binding. However, increase in chloramine $\mathrm{T}$ concentration resulted in decrease of affinity. In a study by Burczynski et al. (1995), palmitate 
exhibits smaller affinity to one of the binding site of albumin oxidized by S-nitrosylation but higher to the other. Other researches showed decrease in binding of phenolsulphophthalein to albumin reduced by nitrosylation (Kashiba-Iwatsuki et al. 1997). Our results show the same dependence in the case of OTA and CPT-C in HSA solution in the presence of chloramine $\mathrm{T}$ (Fig. 4). It is worth to mention that the structure of albumin oxidized by chloramine $\mathrm{T}$ is similar to AOPP isolated from patients (Iwao et al. 2006; Piwowar 2010). Kawasaki et. al. (2006) analyzed the purified HSA from healthy human plasma. They determined the amount of oxidized form of albumin in plasma and then mixed plasma together. They did not analyze binding to albumin for each patient; instead, they oxidized plasma proteins by temperature incubation, cysteine or homocysteine to get plasma with various states of oxidation. This study showed increase in free fraction of 1-Trp and cefazolin in solution of oxidized HSA. While the free fraction of verapamil was found to be slightly decreased. Our study represents a further step towards determining the effect of proteins oxidation on binding drugs to albumin in more physiological conditions, in blood plasma originating from individual healthy patients.

Some research has been also performed with plasma or albumin purified from patients in pathological condition. Study designed by Mera et al. (2005) showed decrease in binding of warfarin to albumin of hemodialysis patients compared to albumin from heathy patients. Similarly, in patients with excretory liver failure, binding affinity of dansylsarcosine decreased in comparison to control subjects (Klammt et al. 2001).

AOPP is a useful tool for measure albumin oxidative damage as well as a general level of oxidative stress. The concentration of AOPP is correlated with the levels of dityrosine and carbonyl groups. Results from our study confirm the connection between level of AOPP and estimated free fraction of OTA and CPT-C in the study group of healthy subjects (Fig. 6). There is a very worthwhile finding because this could be the key in treatment of patients with severe oxidative stress. It is evidence that under conditions of an increased oxidative damage, there is a tendency to decrease in affinity constant and elevate in the free fraction of drugs. Indeed, recently researches showed increase in free fraction of drugs after injection to body in tumor-bearing group of rats (Itakura et al. 2015). The researchers claim that these changes in free fraction are probably involved with oxidative stress in cancer state. Our further studies will be focused on assessing the binding constant of drug to plasma from disease patients.

Summarizing, the results presented in this paper allow to draw a conclusion that the determining of AOPP may be the basis for developing therapeutic strategies (e.g. the appropriate individual dose of drug) for people exposed to strong oxidative stress. An agreement of the obtained results with accessible literature data shows that fluorescence anisotropy method is a reliable and competitive method to other, commonly used techniques.

\section{References}

Akhlaghi F, Ashley JJ, Keogh AM, Brown KF (1998): Indirect esAkhlaghi F, Ashley JJ, Keogh AM, Brown KF (1998): Indirect estimation of the unbound fraction of cyclosporine in plasma. Ther. Drug Monit. 20, 301-308 https://doi.org/10.1097/00007691-199806000-00010

Anraku M, Yamasaki K, Maruyama T, Kragh-Hansen U, Otagiri M (2001): Effect of oxidative stress on the structure and function of human serum albumin. Pharm. Res. 18, 632-639 https://doi.org/10.1023/A:1011029226072

Anraku M, Kragh-Hansen U, Kawai K, Maruyama T, Yamasaki Y, Takakura Y, Otagiri M (2003): Validation of the Chloramine $\mathrm{T}$ induced oxidation of human serum albumin as a model for oxidative damage in vivo. Pharm. Res. 20, 684-692 https://doi.org/10.1023/A:1023219420935

Anraku M, Kitamura K, Shinohara A, Adachi M, Suenaga A, Maruyama T, Miyanaka K, Miyoshi T, Shiraishi N, Nonoguchi $\mathrm{H}$, et al. (2004): Intravenous iron administration induces oxidation of serum albumin in hemodialysis patients. Kidney Int. 66, 841-848 https://doi.org/10.1111/j.1523-1755.2004.00813.x

Berlett BS, Stadtman ER (1997): Protein oxidation in aging, disease, and oxidative stress. J. Biol. Chem. 272, 20313-20316 https://doi.org/10.1074/jbc.272.33.20313

Burczynski FJ, Wang GQ, Hnatowich M (1995): Effect of nitric oxide on albumin-palmitate binding. Biochem. Pharmacol. 49, 91-96 https://doi.org/10.1016/0006-2952(94)00448-U

Burke TG, Mi Z (1994): The structural basis of camptothecin interactions with human serum albumin: impact on drug stability. J. Med. Chem. 37, 40-46 https://doi.org/10.1021/jm00027a005

Cohen LH (2004): Plasma protein-binding methods in drug discovery. In: Methods in Pharmacology and Toxicology, Optimization in Drug Discovery In Vitro Methods (Eds. Z Yan, GW Caldwell), pp. 111-122, Humana Press INC, Totowa, NJ https://doi.org/10.1385/1-59259-800-5:111

Dasgupta A (2008): Handbook of Drug Monitoring Methods: Therapeutics and Drugs of Abuse. Humana Press INC, Totowa, NJ https://doi.org/10.1007/978-1-59745-031-7

Hanasand M, Omdal R, Norheim KB, Gøransson LG, Brede C, Jonsson G (2012): Improved detection of advanced oxidation protein products in plasma. Clin. Chim. Acta 413, 901-906 https://doi.org/10.1016/j.cca.2012.01.038

Itakura Y, Taga S, Iwata C, Teramachi H, Miyamoto K, Tsuchiya H, Wada T, Matsushita R (2015): Structural changes in albumin are a possible mechanism for fluctuation of cefazorin and ibuprofen plasma protein binding in rats with carcinogen-induced osteosarcoma. Anticancer Res. 35, 2063-2069

Iwao Y, Anraku M, Hiraike M, Kawai K, Nakajou K, Kai T, Suenaga A, Otagiri M (2006): The structural and pharmacokinetic 
properties of oxidized human serum albumin, advanced oxidation protein products (AOPP). Drug Metab. Pharmacokinet. 21, 140-146 https://doi.org/10.2133/dmpk.21.140

Jameson DM, Ross JA (2010): Fluorescence polarization/anisotropy in diagnostics and imaging. Chem. Rev. 110, 2685-2708 https://doi.org/10.1021/cr900267p

Kashiba-Iwatsuki M, Miyamoto M, Inoue M (1997): Effect of nitric oxide on the ligand-binding activity of albumin. Arch. Biochem. Biophys. 345, 237-242 https://doi.org/10.1006/abbi.1997.0258

Kawakami A, Kubota K, Yamada N, Tagami U, Takehana K, Sonaka I, Suzuki E, Klammt S, Brinkmann B, Mitzner S, et al. (2001): Albumin binding capacity $(\mathrm{ABiC})$ is reduced in commercially available human serum albumin preparations with stabilizers. Z. Gastroenterol. 39, (Suppl 2), 24-27 https://doi.org/10.1055/s-2001-919056

Klotz IM, Urquhart JM, Weber WW (1950): Penicillin-protein complexes. Arch. Biochem. 26, 420-435

Kober A, Sjöholm I (1980): The Binding Sites on Human Serum Albumin for Some Nonsteroidal Antiinflammatory Drugs. Mol. Pharmacol. 18, 421-426

Kragh-Hansen U, Chuang VTG, Otagiri M (2002): Practical aspects of the ligand-binding and enzymatic properties of human serum albumin. Biol. Pharm. Bull 25, 695-704 https://doi.org/10.1248/bpb.25.695

Krasowski MD, Penrod LE (2012): Clinical decision support of therapeutic drug monitoring of phenytoin: measured versus adjusted phenytoin plasma concentrations. BMC Med. Inform. Decis. Mak. 12, 7 https://doi.org/10.1186/1472-6947-12-7

Kratochwil NA, Huber W, Müller F, Kansy M, Gerber PR (2002): Predicting plasma protein binding of drugs: a new approach. Biochem. Pharmacol. 64, 355-374 https://doi.org/10.1016/S0006-2952(02)01074-2

Kruszewski S, Bom D, Ziomkowska B, Cyrankiewicz M (2006): Affinity of new anticancer agent, DB-174, to membranes and HSA determined by fluorescence spectroscopy methods. Optica Applicata 36, 199-207

Kruszewski S, Ziomkowska B, Cyrankiewicz M, Wybranowski T (2008): The comparison of biophysical properties of DB-67 and its ester DB-67-4ABTFA determined by fluorescence spectroscopy methods. Biosystems 94, 270-275 https://doi.org/10.1016/j.biosystems.2008.05.035

Kurz H, Mauser-Ganshorn A, Stickel HH (1977): Differences in the binding of drugs to plasma proteins from newborn and adult man. I. Eur. J. Clin. Pharmacol. 11, 463-467 https://doi.org/10.1007/BF00562940

Levy RH, Moreland TA (1984): Rationale for monitoring free drug levels. Clin. Pharmacokinet. 9, (Suppl. 1), 1-9 https://doi.org/10.2165/00003088-198400091-00001

Lakowicz JR (1999): Principles of Fluorescence Spectroscopy. Kluwer Academic/Plenum Publishers, New York https://doi.org/10.1007/978-1-4757-3061-6

Mera K, Anraku M, Kitamura K, Nakajou K, Maruyama T, Otagiri M (2005): The structure and function of oxidized albumin in hemodialysis patients: its role in elevated oxidative stress via neutrophil burst. Biochem. Biophys, Res. Commun. 334, 1322-1328 https://doi.org/10.1016/j.bbrc.2005.07.035

Meucci E, Mordente A, Martorana GE (1991): Metal-catalyzed oxidation of human serum albumin: conformational and functional changes. Implications in protein aging. J. Biol. Chem. 266, 4692-4699

Oettl K, Stauber RE (2007): Physiological and pathological changes in the redox state of human serum albumin critically influence its binding properties. Br. J. Pharmacol. 151, 580-590 https://doi.org/10.1038/sj.bjp.0707251

Piwowar A (2010): Advanced oxidation protein products. Part I. Mechanism of the formation, characteristics and property. Pol. Merkur. Lekarski 28, 166-169

Routledge PA, Lazar JD, Barchowsky A, Stargel WW, Wagner GS, Shand DG (1985): A free lignocaine index as a guide to unbound drug concentration. Br. J. Clin. Pharmacol. 20, 695-698 https://doi.org/10.1111/j.1365-2125.1985.tb05132.x

Sitar ME, Aydin S, Cakatay U (2013): Human serum albumin and its relation with oxidative stress. Clin. Lab. 59, 945-952 https://doi.org/10.7754/Clin.Lab.2012.121115

Sudlow G, Birkett DJ, Wade DN (1975): The characterization of two specific drug binding sites on human serum albumin. Mol. Pharmacol. 11, 824-832

Tompsett R, Schulz S, McDermott W (1945): The relation of protein binding to the pharmacology and antibacterial activity of penicillins X, G, Dihydro F, and K. J. Bacteriol. 53, 581-595

Witko-Sarsat V, Friedlander M, Capeillère-Blandin C, NguyenKhoa T, Nguyen AT, Zingraff J, Jungers P, Descamps-Latscha B (1996): Advanced oxidation protein products as a novel marker of oxidative stress in uremia. Kidney Int. 49, 1304-1313 https://doi.org/10.1038/ki.1996.186

Wright JD, Boudinot FD, Ujhelyi MR (1996): Measurement and analysis of unbound drug concentrations. Clin. Pharm. 30, $445-462$ https://doi.org/10.2165/00003088-199630060-00003

Wybranowski T, Cyrankiewicz M, Ziomkowska B, Kruszewski S (2008): The HSA affinity of warfarin and flurbiprofen determined by fluorescence anisotropy measurements of camptothecin. Biosystems 94, 258-262

https://doi.org/10.1016/j.biosystems.2008.05.034

Wybranowski T, Ziomkowska B, Cwynar A, Kruszewski S (2014): The influence of displacement compounds on the binding of ochratoxin A to human serum albumin examined with fluorescence anisotropy methods. Opt. Appl. 44, 357-364

Wörner W, Pfleiderer S, Kratzer W, Rietbrock N (1990): Nonenzymatic glycation of human serum albumin: influence on the binding kinetics of the benzodiazepine binding site. J. Clin. Clin. Biochem. 28, 527-53

Received: December 12, 2017

Final version accepted: May 22, 2018

First published online: October 19, 2018 